\title{
Steiner's invariants and minimal connections
}

\author{
Stephen Ducret and Marc Troyanov \\ (Communicated by Rui Loja Fernandes)
}

\begin{abstract}
The aim of this note is to prove that any compact metric space can be made connected at a minimal cost, where the cost is taken to be the one-dimensional Hausdorff measure.
\end{abstract}

Mathematics Subject Classification (2000). 51Kxx.

Keywords. Metric geometry, Steiner invariant.

\section{Introduction}

Recall that a continuum is a compact connected metric space. We denote by $\operatorname{Cont}(X)$ the set of all continua $C \subset X$ in an arbitrary metric space $(X, d)$.

Definition. (A) Given a metric space $(X, d)$, and a compact subset $S \subset X$, the relative Steiner invariant of $S$ in $X$ is defined as

$$
\operatorname{St}(S, X)=\inf \left\{\mathscr{H}^{1}(C) \mid C \text { and } C \cup S \in \operatorname{Cont}(X)\right\},
$$

here, $\mathscr{H}^{1}$ is the one-dimensional Hausdorff measure.

(B) If $(S, d)$ is a compact metric space, its absolute Steiner invariant is defined as

$$
\begin{gathered}
\operatorname{St}(S)=\inf \{\operatorname{St}(\imath(S), X) \mid X \text { is an arbitrary metric space and } \\
l: S \hookrightarrow X \text { is an isometric embedding }\} .
\end{gathered}
$$

Our goal is to prove the following result.

Theorem 1 (The main theorem). Let $S$ be a compact metric space such that $\operatorname{St}(S)<\infty$. Then its Steiner invariant is realized, i.e., there exist a compact metric space $Z$ and an isometric embedding $\imath: S \hookrightarrow Z$ such that $\operatorname{St}(l(S), Z)=\operatorname{St}(S)$. Furthermore, there exists $C \in \operatorname{Cont}(Z)$ such that $C \cup \imath(S)$ is also a continuum and $\mathscr{H}^{1}(C)=\operatorname{St}(S)$. 
The set $C \cup t(S)$ described in this theorem is thus a "minimal connection" of $S$, i.e., it is a shortest possible set that can be added to $S$ to make it a continuum.

It should be noted that the "Steiner Problem", i.e., the problem of algorithmically finding a minimal connection of a given (generally finite) set in the Euclidean space or other metric spaces such as graphs, has attracted a considerable interest in the literature on optimization and combinatorial geometry, see e.g. [3], [4], [6]. Let us shortly discuss two elementary examples.
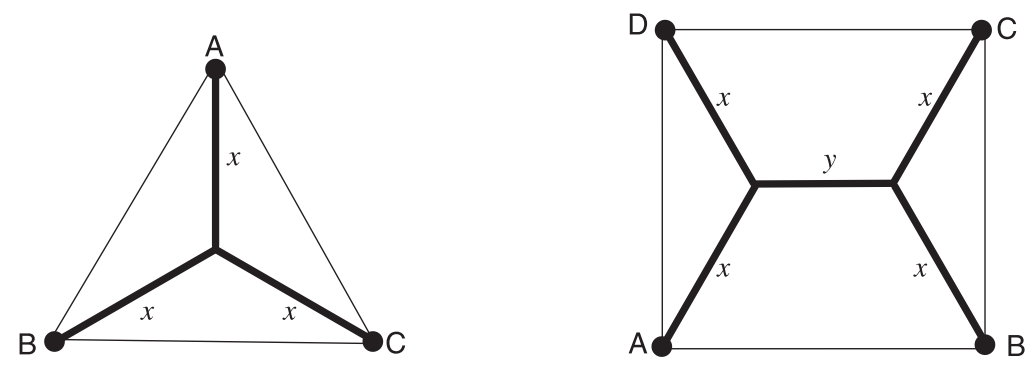

Let $X=\mathbb{R}^{2}$ be the Euclidean space and $S=\{A, B, C\} \subset \mathbb{R}^{2}$ be the three vertices of an equilateral triangle with unit side. Connecting $A, B$ and $C$ to the center of the triangle gives us a trivalent graph whose total length is $3 x$, where $x=1 /(2 \cos (\pi / 6))=\sqrt{3} / 3$. It is known that this graph is the shortest network connecting $A, B$ and $C$ and thus $\operatorname{St}\left(S, \mathbb{R}^{2}\right)=3 x=\sqrt{3}$.

However, the absolute Steiner invariant of that set is smaller. Indeed, one can consider the graph as an abstract metric space. Since $d(A, B)=d(A, C)=$ $d(B, C)=1$, all edges must have length $x^{\prime}=\frac{1}{2}$ and the absolute Steiner invariant of our set is $\operatorname{St}(S)=3 x^{\prime}=\frac{3}{2}<\sqrt{3}$.

As a second example, consider the set $T=\{A, B, C, D\} \subset \mathbb{R}^{2}$ given by the four vertices of a square with unit side. It is known that the shortest network connecting $A, D$ and $B, C$ is a trivalent tree with rectilinear edges forming $120^{\circ}$ angles. From this sole information, one can deduce the shape of that network and compute the lengths. Since $d(A, D)=1$, we have as before $x=\sqrt{3} / 3$, we then compute that $y=1-\sqrt{3} / 3$ and we obtain $\operatorname{St}\left(T, \mathbb{R}^{2}\right)=4 x+y=1+\sqrt{3}$.

Again, one may consider the network as an abstract metric space. Since $d(A, D)=2 x^{\prime}=1$ the exterior edges have length $x^{\prime}=\frac{1}{2}$, and since $d(A, C)=$ $2 x^{\prime}+y^{\prime}=\sqrt{2}$, the interior edge has length $y^{\prime}=\sqrt{2}-1$. The absolute Steiner invariant of that set is then $\operatorname{St}(T)=4 x^{\prime}+y^{\prime}=1+\sqrt{2}<1+\sqrt{3}$.

\section{Useful results}

It is known that the relative Steiner invariant is always realized in a proper metric space (recall that a metric space is proper if every closed ball in it is compact): 
Theorem 2. Let $(X, d)$ be a proper metric space and $S \subset X$ a compact subset such that $\operatorname{St}(S, X)<\infty$. Then $\operatorname{St}(S, X)$ is realized, i.e., there exists $C \in \operatorname{Cont}(X)$ such that $C \cup S \in \operatorname{Cont}(X)$ and $\mathscr{H}^{1}(C)=\operatorname{St}(S, X)$.

If $S$ contains only two points $\{x, y\}$, then this theorem simply says that the two points can be joined by a shortest curve. This is the Hopf-Rinow theorem for proper metric spaces.

This result can be found in [1], Theorem 4.4.20, (see also [4], chapter 2, for the special case of a finite set in a complete Riemannian manifold). The proof is essentially based on the Blaschke compactness theorem for the Hausdorff distance and a semi-continuity property of the Hausdorff measure due to Golab. Let us recall these results.

Proposition 3 (Blaschke). Let $(X, d)$ be an arbitrary metric space. We denote by $\mathscr{K}(X)$ the family of all non empty compact subsets of $X$. This is a metric space for the Hausdorff distance $d_{H}$. We then have:

a) If $(X, d)$ is compact, then so is $\left(\mathscr{K}(X), d_{H}\right)$.

b) If $(X, d)$ is proper, then so is $\left(\mathscr{K}(X), d_{H}\right)$.

This theorem has been originally proved by Blaschke in the context of convex bodies in Euclidean space. We refer to [1], Theorem 4.4.15, or [2], Theorem 7.3.8, for a modern proof.

It is not difficult to check that $\operatorname{Cont}(X) \subset \mathscr{K}(X)$ is a closed subset for the topology induced by the Hausdorff distance. Furthermore:

Proposition 4 (Golab). Let $(X, d)$ be a complete metric space and let $\left\{C_{n}\right\} \subset$ $\operatorname{Cont}(X)$ be a sequence of continua such that $C_{n} \rightarrow C$ for the Hausdorff distance. Then $C \in \operatorname{Cont}(X)$ and

$$
\mathscr{H}^{1}(C) \leq \liminf _{n \rightarrow \infty} \mathscr{H}^{1}\left(C_{n}\right) .
$$

See [1], Theorem 4.4.17, for a proof.

Our main theorem is an extension of Theorem 2. In its proof we will need to replace the Hausdorff distance by the Gromov-Hausdorff distance and the Blaschke theorem will be replaced by the Gromov compactness criterion. To recall this criterion, remember that the packing number of the metric space $X$ at mesh $\varepsilon>0$ is the number

$$
\begin{array}{r}
P(X, \varepsilon)=\min \left\{n \mid \text { there exists } x_{1}, \ldots, x_{n} \in X\right. \text { such that } \\
\text { if } \left.i \neq j \text { then } B\left(x_{i}, \varepsilon\right) \cap B\left(x_{j}, \varepsilon\right)=\emptyset\right\} .
\end{array}
$$

Recall that metric space $X$ is totally bounded if $P(X, \varepsilon)<\infty$ for every $\varepsilon>0$. The Gromov compactness criterion says that a family of isometry classes of compact 
metric spaces is totally bounded for the Gromov-Hausdorff distance if and only if it is uniformly totally bounded:

Theorem 5 (Gromov). Let $\mathscr{M}$ be a family of isometry classes of compact metric spaces. Then the following conditions are equivalent:

i) $\mathscr{M}$ is totally bounded for the Gromov-Hausdorff distance.

ii) $\sup _{X \in \mathscr{M}} P(X, \varepsilon)<\infty$ for any $\varepsilon>0$.

See [2], Theorem 7.4.15.

Another useful result on the Gromov-Hausdorff distance says that any sequence of compact metric spaces, which is Cauchy for the Gromov-Hausdorff distance, contains a subsequence which can be realized as a sequence of subsets of a single compact metric space:

Proposition 6. Let $\left\{X_{n}\right\}$ be a sequence of compact metric spaces which is a Cauchy sequence in the Gromov-Hausdorff sense. Then there exist a subsequence $\left\{X_{n^{\prime}}\right\}$, a compact metric space $Z$ and isometric embeddings $X_{n^{\prime}} \hookrightarrow Y_{n^{\prime}} \subset Z$ and $X \hookrightarrow Y \subset Z$ such that $Y_{n^{\prime}} \rightarrow Y$ for the Hausdorff distance in $Z$.

This result is Theorem 4.5.7 in [1].

\section{Proof of the main theorem}

We first need a lemma:

Lemma 7. Let $(X, d)$ be a compact metric space, and $C \in \operatorname{Cont}(X)$. Then for any $a \in C$ and $0<\varepsilon<\operatorname{diam}(C) / 2$, we have

$$
\mathscr{H}^{1}(C \cap B(a, \varepsilon)) \geq \varepsilon
$$

in particular, we have

$$
P(C, \varepsilon) \leq \frac{1}{\varepsilon} \mathscr{H}^{1}(C) .
$$

The proof of this result can be found for instance in [1], Lemma 4.4.5.

We then need the following generalization of Golab's semi-continuity result:

Proposition 8. Let $\left\{X_{n}\right\}$ be a sequence of compact metric spaces such that $X_{n} \rightarrow X$ in the Gromov-Hausdorff sense. Suppose that $X_{n}$ is connected for each $n$. Then $X$ is compact and connected, and moreover,

$$
\mathscr{H}^{1}(X) \leq \liminf _{n \rightarrow \infty} \mathscr{H}^{1}\left(X_{n}\right) .
$$


Proof. From Proposition 6, we know that, choosing a subsequence if necessary, there exist a compact metric space $Z$ and isometric copies of $X_{n}$ and $X$ embedded in $Z$, say $Y_{n}, Y$, such that $Y_{n} \rightarrow Y$ in the Hausdorff sense. Since $X_{n}$ and $Y_{n}$ are isometric and each $Y_{n}$ is compact and connected, we deduce from Proposition 4 that $Y \in \operatorname{Cont}(Z)$ and

$$
\mathscr{H}^{1}(Y) \leq \liminf _{n \rightarrow \infty} \mathscr{H}^{1}\left(Y_{n}\right) .
$$

Now since $Y$ is isometric to $X$, we conclude that $X$ is compact and connected as well, and that

$$
\mathscr{H}^{1}(X) \leq \liminf _{n \rightarrow \infty} \mathscr{H}^{1}\left(X_{n}\right),
$$

because $\mathscr{H}^{1}\left(X_{n}\right)=\mathscr{H}^{1}\left(Y_{n}\right)$ and $\mathscr{H}^{1}(X)=\mathscr{H}^{1}(Y)$.

Proof of Theorem 1. Let $S$ be a compact metric space, and let $\left\{\left(S_{k}, X_{k}\right)\right\}$ be a minimizing sequence for the absolute Steiner invariant of $S$, that is:

i) $X_{k}$ is a compact metric space, and $S_{k} \subset X_{k}$ is an isometric copy of $S$;

ii) $\mathrm{St}\left(S_{k}, X_{k}\right) \rightarrow \mathrm{St}(S)$.

We first prove that the sequence $\left\{X_{k}\right\}$ can be assumed to be uniformly totally bounded: indeed, if this where not the case, we could replace $X_{k}$ by $S_{k} \cup C_{k}$, where $C_{k} \in \operatorname{Cont}\left(X_{k}\right)$ realizes $\operatorname{St}\left(S_{k}, X_{k}\right)$ (such a set exists by Theorem 2). From the compactness of $S$, we know that $P\left(S_{k}, \varepsilon\right)=P(S, \varepsilon)<\infty$ for all $k$ and all $\varepsilon$. Moreover, $\mathscr{H}^{1}\left(C_{k}\right)=\operatorname{St}\left(S_{k}, X_{k}\right)$, therefore $\sup _{k} \mathscr{H}^{1}\left(C_{k}\right)<\infty$ and the family $\left\{C_{k}\right\}$ is uniformly totally bounded by Lemma 7 . The families $\left\{S_{k}\right\}$ and $\left\{C_{k}\right\}$ being uniformly totally bounded, so is $\left\{S_{k} \cup C_{k}\right\}$.

We henceforth assume $\left\{X_{k}\right\}$ to be uniformly totally bounded. By the Gromov compactness criterion, Theorem 5, we know that $\left\{X_{k}\right\}$ contains a subsequence which is Cauchy in the Gromov-Hausdorff distance. From Proposition 6, we can further take a subsequence which can globally be embedded in a compact metric space $Z$. Finally, using the Blaschke compactness theorem, we can take one more subsequence, which converges for the Hausdorff distance in $Z$.

To sum up, there exist a subsequence $\left\{X_{k^{\prime}}\right\}$, a compact metric space $Z$ and isometric embeddings $l_{k^{\prime}}: X_{k^{\prime}} \hookrightarrow Y_{k^{\prime}} \subset Z$ and a subset $Y \subset Z$ such that $Y_{k^{\prime}} \rightarrow Y$ for the Hausdorff distance in $Z$.

Let $T_{k^{\prime}}=l_{k^{\prime}}\left(S_{k^{\prime}}\right) \subset Y_{k^{\prime}} \subset Z$. By Theorem 2, we can find $C_{k^{\prime}} \in \operatorname{Cont}\left(Y_{k^{\prime}}\right)$ such that $C_{k^{\prime}} \cup T_{k^{\prime}} \in \operatorname{Cont}\left(Y_{k^{\prime}}\right)$ and $\mathscr{H}^{1}\left(C_{k^{\prime}}\right)=\operatorname{St}\left(T_{k^{\prime}}, Y_{k^{\prime}}\right)=\operatorname{St}\left(S_{k^{\prime}}, X_{k^{\prime}}\right)$.

By Blaschke's theorem again, we may assume (taking once more a subsequence if needed) that $\left\{C_{k^{\prime}}\right\}$ converges to a subset $C \subset Z$ for the Hausdorff distance in $Z$. Likewise, we may assume that $\left\{T_{k^{\prime}}\right\}$ converges to a subset $T \subset Z$ (since $C_{k^{\prime}} \cup T_{k^{\prime}} \subset Y_{k^{\prime}}$ and $Y_{k^{\prime}} \rightarrow Y$, we have in fact $C \cup T \subset Y$ ). 
Furthermore, we know from Proposition 4 that $C$ and $C \cup T$ are continua and that $\mathscr{H}^{1}(C) \leq \liminf _{k^{\prime} \rightarrow \infty} \mathscr{H}^{1}\left(C_{k^{\prime}}\right)$. But we have $\mathscr{H}^{1}\left(C_{k^{\prime}}\right)=\operatorname{St}\left(T_{k^{\prime}}, Y_{k^{\prime}}\right)=$ $\operatorname{St}\left(S_{k^{\prime}}, X_{k^{\prime}}\right)$, which converges to $\operatorname{St}(S)$. Thus

$$
\mathscr{H}^{1}(C) \leq \mathrm{St}(S) .
$$

On the other hand $C$ and $C \cup T$ are continua, hence $\mathscr{H}^{1}(C) \geq \operatorname{St}(T)=\operatorname{St}(S)$ by definition of the Steiner invariant. We therefore have equality.

To sum up, we have found a pair of subsets $C, T \subset Z$ such that $C$ and $C \cup T$ are continua, $T$ is isometric to $S$ and $\mathscr{H}^{1}(C)=\operatorname{St}(S)$. The proof is complete.

\section{References}

[1] L. Ambrosio and P. Tilli, Topics on analysis in metric spaces. Oxford Lecture Ser. Math. Appl. 25, Oxford University Press, Oxford 2004. Zbl 1080.28001 MR 2039660

[2] D. Burago, Y. Burago, and S. Ivanov, A course in metric geometry. Grad. Stud. Math. 33, Amer. Math. Soc., Providence, RI, 2001. Zbl 0981.51016 MR 1835418

[3] F. K. Hwang, D. Richards, and P. Winter, The Steiner tree problem. Ann. Discrete Math. 53, North-Holland, Amsterdam 1992. Zbl 0774.05001 MR 1192785

[4] A. Ivanov and A. Tuzhilin, Minimal networks: the Steiner problem and its generalizations. CRC Press, Boca Raton 1994. Zbl 0842.90116 MR 1271779

[5] A. Papadopoulos, Metric spaces, convexity and nonpositive curvature. IRMA Lect. Math. Theor. Phys. 6, Eur. Math. Soc. Publ. House, Zürich. Zurich 2005.

Zbl 1115.53002 MR 2132506

[6] H. Prömel and A. Steger, The Steiner tree problem. Adv. Lectures Math., Vieweg, Wiesbaden 2002. Zbl 0992.68156 MR 1891564

Received January 16, 2007; revised February 15, 2007

S. Ducret, Section de Mathématiques-IGAT, École Polytechnique Féderale de Lausanne, 1015 Lausanne, Switzerland

E-mail: stephen.ducret@epfl.ch

M. Troyanov, Section de Mathématiques-IGAT, École Polytechnique Féderale de Lausanne, 1015 Lausanne, Switzerland

E-mail: marc.troyanov@epfl.ch 